\title{
Spectral interferometric technique to measure the relative phase change on reflection from a thin-film structure
}

\author{
P. Hlubina, D. Ciprian, J. Luňáček \\ Department of Physics, Technical University Ostrava, 17. listopadu 15, 70833 Ostrava-Poruba, Czech Republic
}

Received:

\begin{abstract}
A two-step white-light spectral interferometric technique to measure the relative phase change on reflection from a thin-film structure is presented. The technique is based on recording of the channelled spectra at the output of a Michelson interferometer and their processing by using a windowed Fourier transform to retrieve the phase functions. In the first step, the phase difference between the beams of the interferometer with a thin-film structure is retrieved. In the second step, the structure is replaced by a reference sample of known phase change on reflection and the corresponding phase difference is retrieved. From the two phase differences, the relative phase change on reflection from the thinfilm structure is obtained. The feasibility of the simple method is confirmed in processing the experimental data for a $\mathrm{SiO}_{2}$ thin film on a $\mathrm{Si}$ wafer of known optical constants. Four samples of the thin film are used and their thicknesses are determined. The thicknesses obtained are compared with those resulting from reflectometric measurements, and good agreement is confirmed.
\end{abstract}

PACS 07.60.Ly, 42.30.Rx, 68.55.Jk

\section{Introduction}

Optical methods, based on ellipsometric [1,2], reflectometric [3] or interferometric [4-6] measurements, belong to the most important ones in research of thin-film structures. Ellipsometric angles, reflectances and interferometric phases are crucial in determining the parameters and characteristics of structures. Ellipsometric measurements performed at a single wavelength and a fixed angle of incidence provide the film thickness and optical constants [1]. Measurements by spectroscopic ellipsometry provide the results over a wide wavelength range

Correspondence to: P. Hlubina, Phone: 420597323 134,

Fax: 420597323 403, E-mail: petr.hlubina@vsb.cz with greater precision and accuracy [2]. Normal incidence spectroscopic reflectometry [3] applied over a wide wavelength range is also a useful tool for the characterization of thin films. The optical method most commonly employed for micrometer-scale thickness measurements is Fourier transform infrared [4] and white-light [5] interferometry. The latter technique is based on retrieving the relative phase change on reflection from a thin film. The use of white-light interferometry was extended into the spectral domain $[6,7]$ where the phase of the reflected wave, which changes as a function of wavelength [8] and layer thickness, is inscribed in the recorded spectral interferogram (channelled spectrum). Moreover, a spectral-domain phase-shifting interferometric technique is of interest to researchers in retrieving the relative phase change on reflection from a thin film and in determining the film thickness [9].

Recently, we have used dispersive white-light spectral interferometry for measuring the thickness of a $\mathrm{SiO}_{2}$ thin film on a Si wafer [10]. The technique utilizes a slightly dispersive Michelson interferometer with one of the mirrors replaced by a thin-film structure of known optical constants. The thickness of the thin film is determined from the fit of the recorded spectral interferogram to the theoretical one. More recently, the use of dispersive white-light spectral interferometry was extended for measuring the thin-film thickness utilizing the absolute phase retrieval from the spectral interferogram [11]. However, the results suffered from the systematic phase errors due to the optical components present in the interferometer. To minimize them, a procedure with the reference measurement needs to be applied [7]. We applied a three-step procedure [12] that enabled us to determine both the effective thickness of a beam splitter cube and the phase contribution of the interferometer optical elements. Moreover, the spectral reflectance measurement was possible in the same set-up. These techniques have also been used for measuring small changes in the thickness of $\mathrm{a} \mathrm{SiO}_{2}$ thin film grown by thermal oxidation on different Si substrates [13]. 
In this paper, a two-step white-light spectral interferometric technique, which is similar to a two-step reflectometric technique with the reference measurement, is presented to retrieve the relative phase change on reflection from of a thin-film structure. The technique, which represents a substantial simplification of a previous method [12], is based on recording of the channelled spectra at the output of a Michelson interferometer and their processing by using a windowed Fourier transform (WFT) to retrieve the relative phase. In the first step, the phase difference between the beams of the interferometer with a a thin-film structure is retrieved. In the second step, the structure is replaced by a reference sample of known phase change on reflection and the corresponding phase difference is retrieved. From the two phase functions, the relative phase change on reflection from the thinfilm structure is obtained. The feasibility of the simple method was confirmed in processing the experimental data for a $\mathrm{SiO}_{2}$ thin film on a $\mathrm{Si}$ wafer of known optical constants. Four samples of the thin film were used and their thicknesses were determined. The thicknesses obtained were compared with those resulting from reflectometric measurements, and good agreement was confirmed.

\section{Theoretical background}

Consider the mutual interference of two beams from a broadband source at the output of a Michelson interferometer (see Fig. 1) with a cube beam splitter of the effective thickness $t_{\text {eff }}[10]$. The interferometer comprises a mirror and a thin-film structure that are characterized by the complex reflection coefficients

$$
r_{M}(\lambda)=\sqrt{R_{M}(\lambda)} \exp \left[\mathrm{i} \delta_{M}(\lambda)\right]
$$

and

$$
r(\lambda)=\sqrt{R(\lambda)} \exp \left[\mathrm{i} \delta_{r}(\lambda)\right]
$$

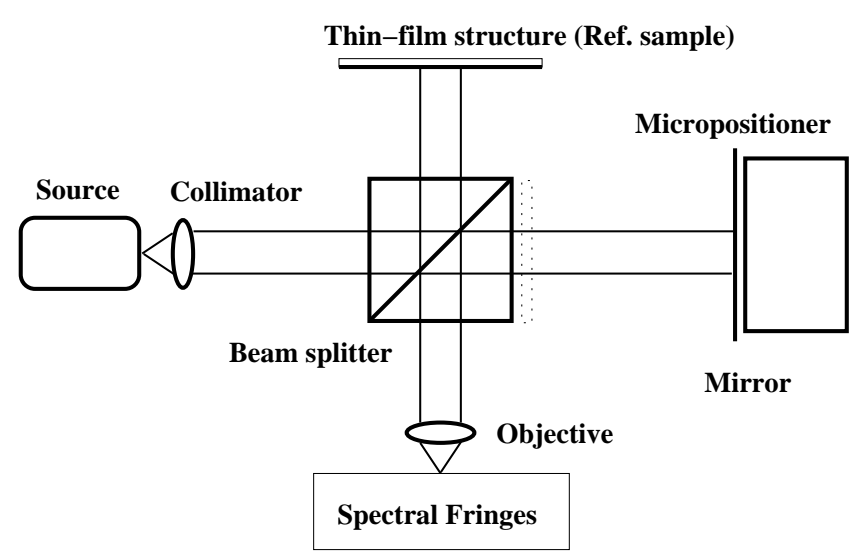

Fig. 1 Experimental set-up with a Michelson interferometer to measure the relative phase change on reflection from a thin-film structure. where $R_{M}(\lambda)$ and $R(\lambda)$ are the wavelength-dependent reflectances of the mirror and the structure, respectively, $\delta_{M}(\lambda)$ and $\delta_{r}(\lambda)$ are the phase changes on reflection from the mirror and the thin-film structure, respectively. We assume that the geometrical path lengths of the light rays in dispersive glass of the beam splitter are not the same for both interferometer arms and the beam splitter is represented by an ideal beam splitter and a plate (see a dashed element in Fig. 1) of the same dispersion and of the thickness $t_{\text {eff }}$. The interference between the beams of the Michelson interferometer is resolved by a spectrometer as the channelled spectrum

$$
I(\lambda)=I_{0}(\lambda)\{1+V(\lambda) \cos [\Delta \phi(\lambda)]\}
$$

where $I_{0}(\lambda)$ is the reference spectrum, $V(\lambda)$ is a visibility term, which depends on the reflectance $R(\lambda)$ of the thin film structure [10], and $\Delta \phi(\lambda)$ is the phase difference given by

$\Delta \phi(\lambda)=\delta_{M}(\lambda)-\delta_{r}(\lambda)-\delta_{B S}(\lambda)+2 \pi\left[2 L_{1}+2 n(\lambda) t_{\text {eff }}\right] / \lambda$

where $\delta_{B S}(\lambda)$ is the phase change owing to the beam splitter, $2 L_{1}$ is the difference of path lengths between the interfering beams in the air whose dispersion is neglected and $n(\lambda)$ is the refractive index of the beam splitter material. The extraction of the phase difference $\Delta \phi(\lambda)$ can be easily achieved in the wavelength domain by applying a WFT [14]. Because the phase difference $\Delta \phi(\lambda)$ given by Eq. (4) contains not only $\delta_{r}(\lambda)$, which is to be retrieved, but also the phase changes introduced by the beam splitter and the mirror, a reference measurement is required for the extraction of $\delta_{r}(\lambda)$.

For the reference measurement, the thin-film structure is replaced by a reference sample of the known complex reflection coefficient

$$
r_{r e f}(\lambda)=\sqrt{R_{r e f}(\lambda)} \exp \left[\mathrm{i} \delta_{r e f}(\lambda)\right]
$$

and the interference between the beams of the Michelson interferometer gives rise to the channelled spectrum

$$
I_{\text {ref }}(\lambda)=I_{\text {oref }}(\lambda)\left\{1+V_{\text {ref }}(\lambda) \cos \left[\Delta \phi_{\text {ref }}(\lambda)\right]\right\},
$$

where $\Delta \phi_{\text {ref }}(\lambda)$ is the phase difference given by

$\Delta \phi_{\text {ref }}(\lambda)=\delta_{M}(\lambda)-\delta_{\text {ref }}(\lambda)-\delta_{B S}(\lambda)+2 \pi\left[2 L_{2}+2 n(\lambda) t_{\text {eff }}\right] / \lambda$

The relative phase change on the reflection from the thin-film structure can be obtained as

$$
\delta_{r}(\lambda)=\delta_{r}(\lambda ; \Delta L)-4 \pi \Delta L / \lambda
$$

where

$$
\delta_{r}(\lambda ; \Delta L)=\Delta \phi_{\text {ref }}(\lambda)-\Delta \phi(\lambda)+\delta_{\text {ref }}(\lambda)
$$

is the measured phase change retrieved from the two recorded channelled spectra and $\Delta L=L_{2}-L_{1}$. It should be noted that the retrieved phase $\delta_{r}(\lambda ; \Delta L)$ is not the 
absolute phase change since it can be extracted with an ambiguity of $2 \pi$. Moreover, it is slightly modified with respect to $\delta_{r}(\lambda)$ due to a term $4 \pi \Delta L / \lambda$ since $L_{2}$ slightly different from $L_{1}$ may be adjusted in the interferometer in the second measurement step.

\section{Numerical example}

To demonstrate the applicability of our two-step method in retrieving the relative phase change $\delta_{r}(\lambda ; \Delta L)$ that differs from $\delta_{r}(\lambda)$, we consider light incident on a surface of a thin-film structure represented by a uniform $\mathrm{SiO}_{2}$ thin film of the thickness $d=450 \mathrm{~nm}$ on a Si substrate. In this case multiple reflections take place and the complex reflection coefficient $r(\lambda)$ of the structure can be expressed according to the well-known relation $[1,10]$. To simulate the channelled spectrum $I(\lambda)$ resolvable in the first step, we consider a Michelson interferometer with a cube beam splitter made of pure BK7 optical glass whose effective thickness is $t_{\text {eff }}=-10 \mu \mathrm{m}$. Next, we consider that $\delta_{M}(\lambda)=\delta_{B S}(\lambda)=0$. Taking into account the known wavelength dependences for the refractive index $n(\lambda)$ of the pure BK7 optical glass [15], the refractive index of the $\mathrm{SiO}_{2}$ thin film [10] and the complex refractive index of the $\mathrm{Si}$ substrate [16], we computed the spectrum $I(\lambda)$ in the range from 350 to $1000 \mathrm{~nm}$ for a suitably chosen $L_{1}$. The corresponding spectral signal $S(\lambda)=I(\lambda) / I_{0}(\lambda)-1$ with added noise is shown in Fig. 2 by the solid line. To simulate the reference channelled spectrum $I_{r e f}(\lambda)$ resolvable in the second step, we consider that the thin-film structure in the Michelson interferometer is replaced by a reference sample represented by a bare Si substrate and that such a path length difference $L_{2}$ is adjusted so that $\Delta L=100 \mathrm{~nm}$. The corresponding spectral signal $S_{\text {ref }}(\lambda)=I_{\text {ref }}(\lambda) / I_{0 r e f}(\lambda)-1$ with added noise is shown in Fig. 2 by the dashed line.

The two spectral signals are processed by a WFT [14] to retrieve the unwrapped phase differences $\Delta \phi(\lambda)$ and $\Delta \phi_{r e f}(\lambda)$ with the ambiguity of $m 2 \pi$, where $m$ is an integer. From the phase differences we obtain the relative phase change $\delta_{r}(\lambda ; \Delta L)$, which is shown in Fig. 3 by the solid curve. It is compared with the theoretical relative phase change to determine the thickness $d$ of the $\mathrm{SiO}_{2}$ thin film on the $\mathrm{Si}$ substrate. The theoretical relative phase change $\delta_{r}(\lambda ; \Delta L)$ is fitted to the retrieved one $\delta_{r}^{r}(\lambda ; \Delta L)$ using the Levenberg-Marquardt least-squares algorithm [17]. The method determines the maximumlikelihood estimate of parameters $\Delta L$ and $d$ that minimizes the figure-of-merit function $\chi^{2}$, defined by

$$
\chi^{2}(\Delta L, d)=\sum_{i=1}^{N}\left[\delta_{r}^{r}\left(\lambda_{i} ; \Delta L\right)-\delta_{r}\left(\lambda_{i} ; \Delta L, d\right)\right]^{2},
$$

where $\lambda_{i}$ are wavelengths at which the fit is performed (450 to $900 \mathrm{~nm}$ ). We reached an excellent agreement between the theoretical values and the results of processing

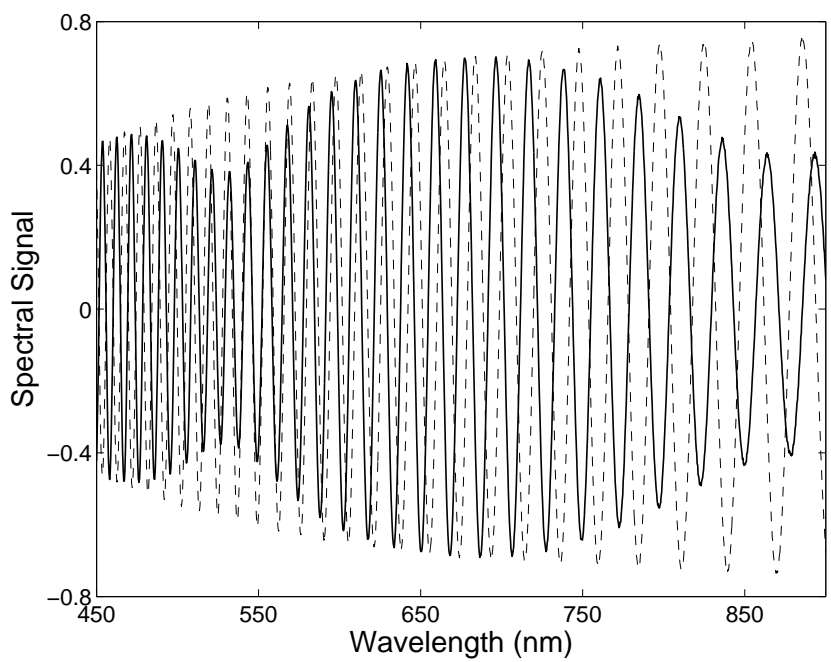

Fig. 2 Theoretical spectral signal with (solid) and without (dashed) the effect of the $\mathrm{SiO}_{2}$ thin film of thickness $d=450 \mathrm{~nm}$ on the Si substrate $(\Delta L=100 \mathrm{~nm})$.

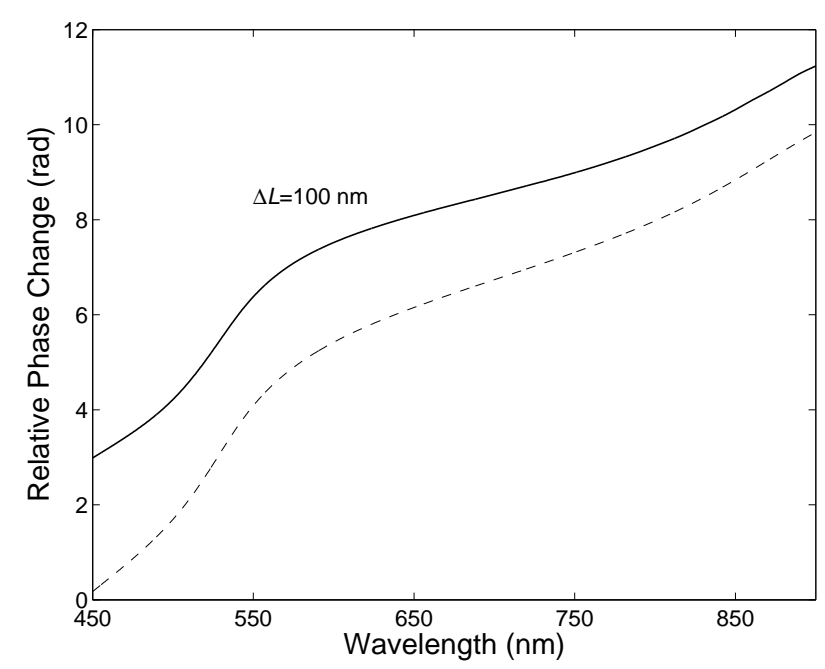

Fig. 3 Relative phase change $\delta_{r}(\lambda ; \Delta L)$ retrieved from the spectral signals shown in Fig. 2. Dashed line is the theoretical function $\delta_{r}(\lambda)$.

(the correlation coefficient is 1 ) with values of two parameters: $\Delta L=100.0 \mathrm{~nm}$ and $d=449.9 \mathrm{~nm}$. In Fig. 3 is also shown the theoretical relative phase change $\delta_{r}(\lambda)$. It is clearly seen from Fig. 3 that the phase changes $\delta_{r}(\lambda ; \Delta L)$ and $\delta_{r}(\lambda)$ are not only shifted but are also slightly rotated each other.

\section{Experimental set-up}

The experimental set-up used in the application of whitelight spectral interferometry to measure the relative phase change on reflection from a thin-film structure is shown in Fig. 1. It consists of a white-light source: a halogen lamp HL-2000 (Ocean Optics) with launching optics, a collimating lens, a bulk-optic Michelson interfer- 
ometer with a cube beam splitter made of BK7 optical glass, a metallic mirror connected to a micropositioner, a thin-film structure, a miniature fibre-optic spectrometer S2000 (Ocean optics) and other components [12]. The thin-film structure is represented by a uniform $\mathrm{SiO}_{2}$ thin film on a Si wafer. Four different samples with four different $\mathrm{SiO}_{2}$ thin-film thicknesses were under study as is specified in a previous paper [12]. The Si wafers were annealed in a furnace at $1200^{\circ} \mathrm{C}$ and four annealing times were selected in order to prepare the $\mathrm{SiO}_{2}$ thin film of four different thicknesses ranging approximately from 300 to $450 \mathrm{~nm}$.

\section{Experimental results and discussion}

In the first step, the channelled spectrum $I(\lambda)$ is recorded at the output of the Michelson interferometer with the thin-film structure under study when the path length difference $L_{1}$ is adjusted. To obtain the spectral signal $S(\lambda)$, which is needed in retrieving the phase difference $\Delta \phi(\lambda)$ by using the WFT, the reference spectrum $I_{0}(\lambda)$, i.e., the spectrum without spectral interference fringes, needs to be recorded. To obtain the reference spectrum, one manually translates the micropositioner until spectral fringes disappear completely.

In the second step, the thin film structure is replaced by a bare Si wafer whose complex reflection coefficient $r_{r e f}(\lambda)$ is known and the channelled spectrum $I_{r e f}(\lambda)$ is recorded at the output of the Michelson interferometer when the path length difference $L_{2}$ is adjusted very close to $L_{1}$. The reference spectrum $I_{0 r e f}(\lambda)$ is also recorded in the set-up. From the corresponding spectral signal $S_{r e f}(\lambda)$, the phase difference $\Delta \phi_{r e f}(\lambda)$ is retrieved. Using the known phase change $\delta_{r e f}(\lambda)$ on reflection from the $\mathrm{Si}$ substrate computed from the data available [16], the measured relative phase $\delta_{r}(\lambda ; \Delta L)$ according to Eq. (9) is obtained. The function was compared with the theoretical one in order to determine the thickness $d$ of different samples of the $\mathrm{SiO}_{2}$ thin film on the $\mathrm{Si}$ substrate.

Figure 4 shows the comparison of the results of fitting the theoretical relative phase change to the measured one for sample 1 using the Levenberg-Marquardt leastsquares algorithm presented above. Figure 4 demonstrates very good agreement between theory and experiment with the correlation coefficient as high as 0.99946, the path length difference $\Delta L=176 \mathrm{~nm}$ and the thin-film thickness $d=283.8 \mathrm{~nm}$. In the same figure is shown the measured relative phase change on reflection from sample 2 compared with the theoretical one. Once again, very good agreement is confirmed with parameters of the fit: the correlation coefficient as high as 0.99923 , the path length difference $\Delta L=285 \mathrm{~nm}$ and the thin-film thickness $d=337.6 \mathrm{~nm}$.

Next, Fig. 5 shows a comparison of the experimental results with the theoretical ones concerning the relative

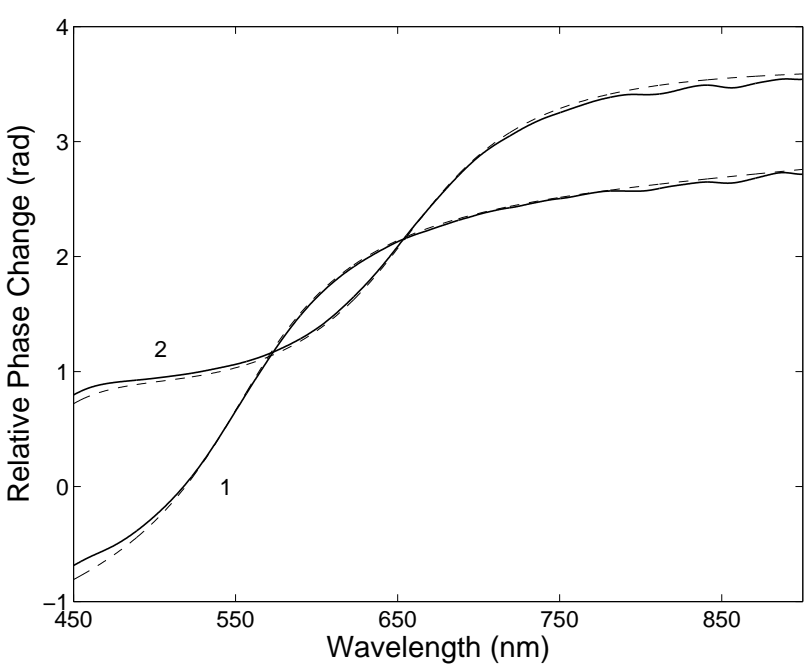

Fig. 4 Measured relative phase change as a function of wavelength with the corresponding fit (dashed) for samples 1 and 2

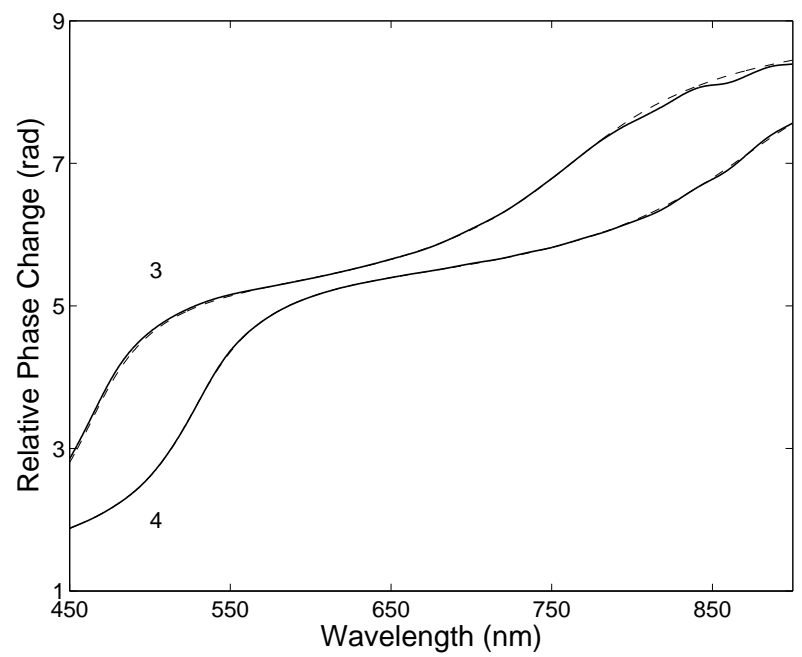

Fig. 5 Measured relative phase change as a function of wavelength with the corresponding fit (dashed) for samples 3 and 4

phase change $\delta_{r}(\lambda ; \Delta L)$ for the remaining two samples. Figure 5 illustrates very good agreement between theory and experiment and Table 1 lists the corresponding thicknesses $d$ and the correlation coefficients R. We measured also in the same set-up the reflectances of the samples by using a three-step procedure presented in a previous paper [12] and Table 1 lists the thicknesses $d_{R}$ obtained.

We can conclude from the obtained results that there is very good agreement between experiment and theory. The correlation coefficients $\mathrm{R}$ indicate minimum discrepancy between the real structure and the theoretical model adopted. The correlation coefficients $\mathrm{R}$ show that the best agreement is reached for the fourth sample and the worst for the second one. Moreover, the results ob- 
Table 1 The oxidation time $T$ and the thicknesses $d_{R}[12]$ and $d$ of the $\mathrm{SiO}_{2}$ thin films ( $\mathrm{R}$ is the correlation coefficient)

\begin{tabular}{ccccc}
\hline Sample & $T(\min )$ & $d_{R}(\mathrm{~nm})$ & $d(\mathrm{~nm})$ & $\mathrm{R}$ \\
\hline 1 & 122 & 285.3 & 283.8 & 0.99946 \\
2 & 212 & 337.4 & 337.6 & 0.99923 \\
3 & 326 & 390.2 & 393.2 & 0.99964 \\
4 & 392 & 450.6 & 449.4 & 0.99997 \\
\hline
\end{tabular}

tained in the same set-up by techniques of interferometry and reflectometry agree well.

\section{Conclusions}

We used a simple two-step method for a precise measurement of the relative phase change on reflection from a thin-film structure. The method is based on white-light spectral interferometry and uses processing of recorded channelled spectra to extract phase functions. Two steps, one with a sample under study and the other one with a reference sample of known phase change on reflection, allow one to eliminate the phase errors due to optical elements of the interferometer. Moreover, in the two steps the phase functions are obtained without the knowledge of the effective thickness of a beam splitter cube, what substantially simplifies the retrieval of the measured relative phase change compared to a previous method [12].

The feasibility of the method was confirmed in measuring the relative phase change on reflection from a $\mathrm{SiO}_{2}$ thin film on a $\mathrm{Si}$ wafer. The measured relative phase changes were compared with theoretical ones to determine the thin-film thickness for four samples. We confirmed very good agreement between the theoretical and experimental phase changes and between the thicknesses determined in the same set-up from interferometric and reflectometric measurements.

The results obtained serve as an illustration of the feasibility of a simple technique in measuring precisely the relative phase change on reflection from a thin-film structure. This method has the primary advantage in its normal incidence configuration over a technique such as ellipsometry. It has potential applications in the phase characterization of complex structures such as multilayer mirrors [18]. This technique and a technique of normal incidence reflectometry, applicable in the same set-up, can serve as complements to spectroscopic ellipsometry.

Acknowledgements The research has been partially supported by the Ministry of Education, Youth and Sports of the Czech Republic through grant MSM6198910016 and by the regional grant CZ.1.05/2.1.00/01.0040.

\section{References}

1. R. A. Azzam, N. M. Bashara, Ellipsometry and Polarized Light (North-Holland, Amsterdam 1977)

2. G. E. Jellison, Jr., Thin Solid Films 290-291, 40 (1996)

3. R. Swanepoel, J. Opt. Soc. Am. A 2, 1339 (1985)

4. M. Kildemo, V. Dalsrud, O. Fostad, Opt. Eng. 38, 1542 (1999)

5. S.-W. Kim, G.-H. Kim, Appl. Opt. 38, 5968 (1999)

6. U. Schnell, R. Dändliker, S. Gray, Opt. Lett. 21, 528 (1996)

7. Y.-S. Ghim, S.-W. Kim, Appl. Phys. Lett. 91, 091903 (2007)

8. T. Doi, K. Toyoda, Y. Tanimura, Appl. Opt. 36, 7157 (1997)

9. S. K. Debnath, M. P. Kothiayl, J. Schmit, P. Hariharan, Opt. Express 14, 4662 (2006)

10. P. Hlubina, D. Ciprian, J. Luňáček, M. Lesňák, Appl. Phys. B 84, 511 (2006)

11. P. Hlubina, D. Ciprian, J. Luňáček, M. Lesňák, Opt. Express 14, 7678 (2006)

12. P. Hlubina, J. Luňáček, D. Ciprian, R. Chlebus, Appl. Phys. B 92, 203 (2008)

13. P. Hlubina, J. Luňáček, Appl. Phys. B 95, 795 (2009)

14. P. Hlubina, J. Luňáček, D. Ciprian, R. Chlebus, Opt. Commun. 281, 2349 (2008)

15. Schott Computer Glass Catalog 1.0 (Schott Glasswerke, Mainz 1992)

16. E. D. Palik, Handbook of Optical Constants of Solids (Academic Press, Orlando 1995)

17. Optimization Toolbox for Use with MATLAB (MathWorks, Mass. 2000)

18. P. Hlubina, J. Luňáček, D. Ciprian, Opt. Lett. 34, 1564 (2009) 